\title{
Foreign body granuloma of the penis caused by occupational glass fibre exposure
}

\author{
U Hinnen, P Elsner, M Barraud, G Burg
}

We report a patient who presented with the suspected diagnosis of syphilis. Clinical findings included a penile ulcer, positive history of syphilis more than 20 years ago, and positive syphilis serology (TPHA, FTA-Abs). A biopsy showed a plasma-cell rich inflammation with granuloma formation. Since a birefractory structure was observed in the biopsy possibly corresponding to a foreign body, the patient's occupational exposure was investigated. Working in the fibre reinforced plastics industry, he was heavily exposed to glass fibre that was even detected on the inside of his underwear. Taking the serological pattern into account that was not consistent with active syphilis, a penile ulcer following a foreign body reaction was diagnosed. This case report demonstrates the difficulties of differentiating foreign body granuloma of the genital region from venereal diseases with granuloma formation.

(Genitourin Med 1997;73:577-578)

Keywords: foreign body granuloma; glass fibre

\section{Introduction}

Foreign body granuloma is characterised by a chronic proliferative inflammatory skin reaction. Microscopic examination reveals lymphocytes, histiocytes, macrophages, epithelioid cells, and giant cells. A central necrotic zone with a caseous appearance may be present. ${ }^{1}$ This reaction is caused by substances such as wood, silk, nylon, paraffin, silicon, talc, starch, oils, animal and vegetable spines or bristles, and human hair. ${ }^{1}$ Foreign body reactions caused by glass fibres are also well described. ${ }^{2-4}$ They are caused by the embedding of glass slugs and occur rarely in comparison with the frequent "glass fibre dermatitis" that is accompanied by rapidly healing papules, vesicles, pustules, and excoriations. ${ }^{5}$

A foreign body granuloma localised on the penis may easily be mistaken for a venereal disease with granuloma formation. In order to demonstrate the difficulties in differentiating foreign body granuloma at this location from morphologically similar diseases like stage III syphilis we present the following case.

\section{Case report}

Six months before the presentation at our clinic a 53 year old man developed an ulcer $2 \mathrm{~cm}$ in diameter on the prepuce, which subsequently also involved the glans penis. Because of his history of stage I syphilis which was treated in the 1960s and the clinical finding he was treated by his physician with two shots of benzathine penicillin $(2.4$ million units) intramuscularly, although the serology did not indicate an active disease (VDRL negative, TPHA slightly positive $(1: 80)$, FTA Abs positive). Within the following months the penile ulcers did not heal and the patient developed a scarring phimosis which was surgically treated. A tissue specimen was examined histologically and described as a granuloma, consistent with the diagnosis of syphilis.
At the time the patient was referred to our clinic two indurated, non-ulcerated plaques were present on the penis (fig 1), that had recently been circumcised. Small mobile inguinal lymph nodes were palpable bilaterally. The general examination of the skin was normal. Serologically, he had evidence of past infection, but the tests did not indicate a reactivation of the disease (VDRL negative, TPHA slightly positive (1:160), FTA Abs slightly positive) and remained unchanged, when repeated 2 months later. Chlamydia trachomatis serology (CBR) was negative (1:20). The same was true for HIV testing. A skin

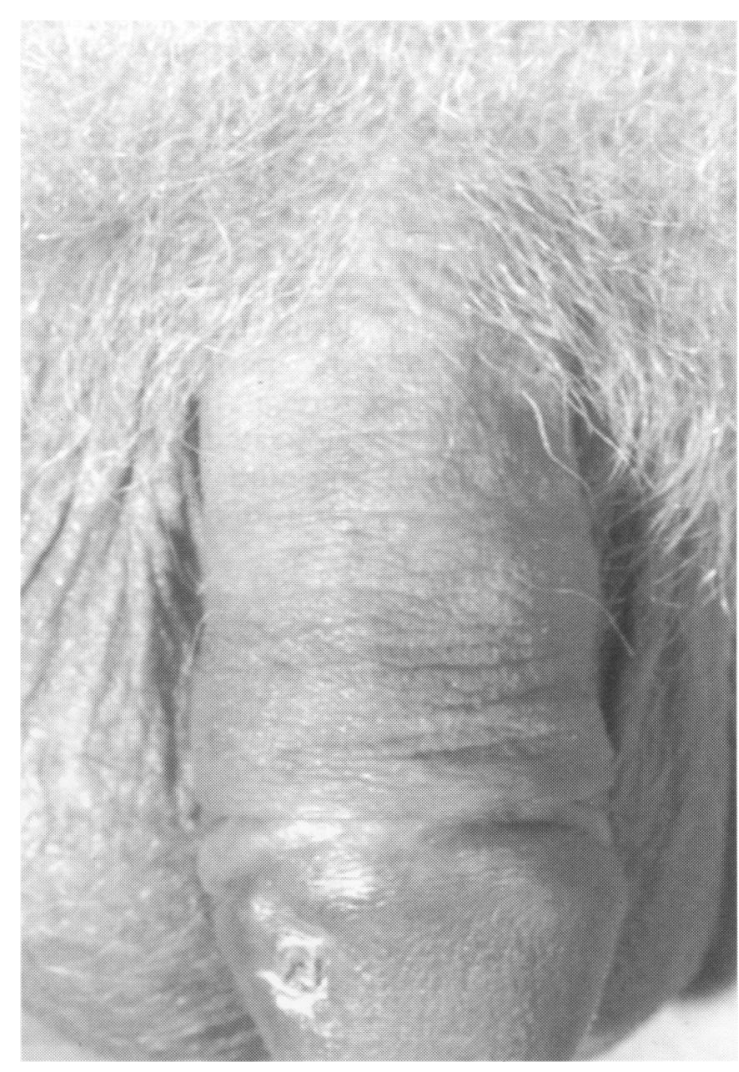

Figure 1 One of the two indurated plaques (at the time the patient presented at the clinic). \\ Department of \\ University of Zurich \\ 8 July 1997
}



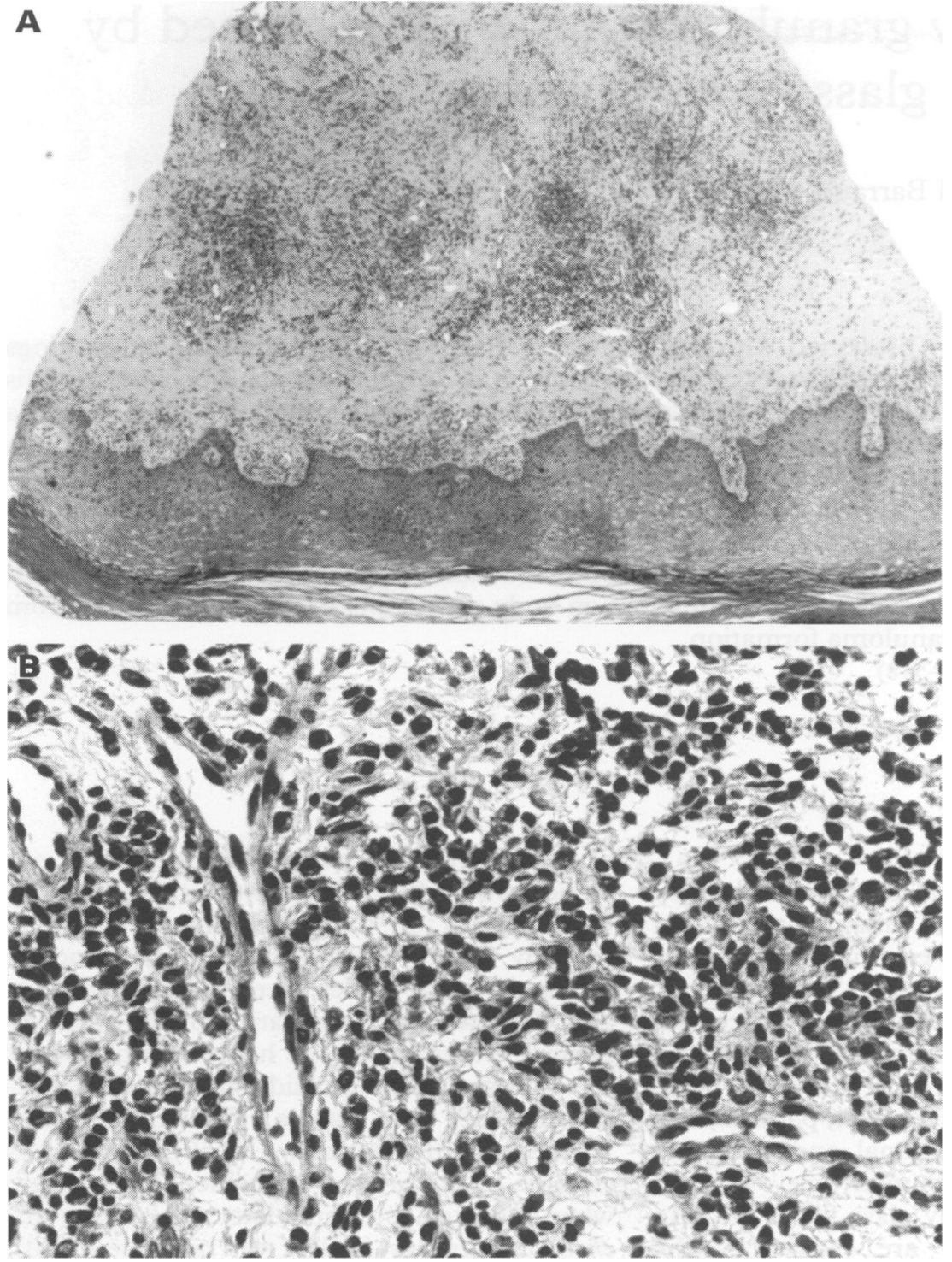

Figure 2(A) Foreign body granuloma of the penis showing a granulomatous lymphohistiocytic infiltrate in the dermis. Haematoxylin and eosin, magnification $\times 21$ (B) Same lesion as $(A)$. Close up view of the mixed cellular infiltrate composed of lymphocytes and histiocytes. Haematoxylin and eosin, magnification $\times 335$.

biopsy of one of the plaques (fig $2 \mathrm{~A}$ and B) showed a diffuse and also perivascular inflammatory infiltrate, consisting mainly of plasma cells. In addition, granulomas were found with degenerative changes at the centre of the lesion and epithelioid as well as giant cells at the periphery. Within the granulomas no foreign bodies were observed. However, an elongated birefractive structure possibly corresponding to a glass fibre was identified in the stratum corneum (fig 3 ). It was concluded that

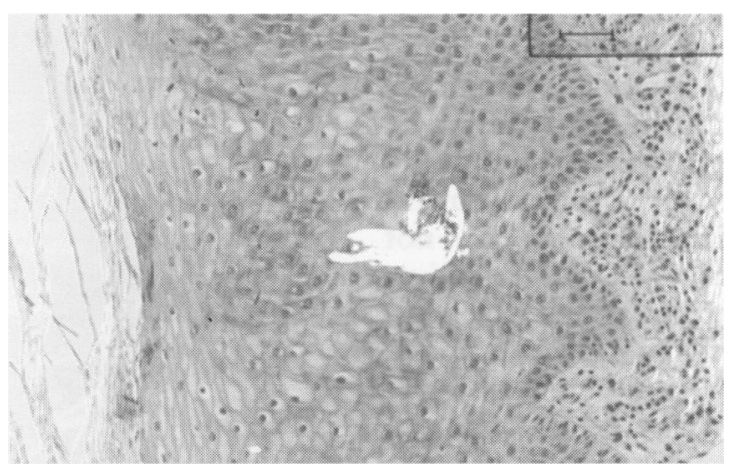

Figure 3 Birefractive glass fibre. Haematoxylin and eosin, magnification $\times 95$. the finding was consistent not only with an inflammatory reaction in late phase syphilis, but also with a granulomatous foreign body reaction and an examination in our occupational dermatology unit was initiated. The occupational history revealed that the patient $\Omega$ started a new job 4 years earlier in a factory $\stackrel{\Phi}{I}$ manufacturing fibre reinforced plastic products. He spent most of his working time cutting up glass fibre mats before they were used 3 for lamination in another part of the plant. $\stackrel{\odot}{?}$ During this activity he was heavily exposed to glass fibres that were released in the air and $\stackrel{5}{+}$ deposited on his clothes and skin. He fre- $\frac{C}{0}$ quently suffered from itching. On the occasion $\frac{\bar{c}}{\mathrm{c}}$ of a visit to the workplace glass fibres were also $\overparen{\nabla}$ identified on the inner side of his underpants although he was trying to stop penetration by ${ }^{\text {c }}$ wearing especially close trousers underneath his overall at the time of the visit.

\section{Discussion}

In our patient exposure to glass fibres was heavy, and fibres were detected on the inner side of his pants after work. Close contact of $\mathrm{O}$ fibres with penile skin was therefore likely. In addition, the histological findings of the skin

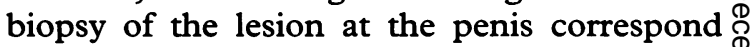
well with a foreign body granuloma. The fact 3 that glass fibres were not detected in the gran- $\mathbb{D}$ ulomas but only in the overlying epidermis does not exclude this diagnosis since foreign bodies may be extremely difficult to trace even on serial sections.

After the patient had been circumcised the lesion healed within a few months, and he developed no new lesions despite unchanged $\stackrel{\square}{\mathscr{Q}}$ exposure. We propose that the lesions only $\stackrel{2}{\vec{T}}$ appeared as long as fibres were deposited between glans and prepuce, where they easily penetrated the moist, occluded skin, and dis-? appeared after the prepuce had been removed. क? Other diagnoses are much less likely. Although the clinical appearance and the histological findings do not allow the possibility of a $\delta$ gumma (with coincidental glass fibre in the stratum corneum) to be completely ruled out, 음 serology strongly argues against it, since only $\rightarrow$ the specific tests (TPHA, FTA Abs) were slightly positive, while the VDRL was negative of at control and 2 months later. The same is $N$ true for Chlamydia trachomatis infection (lym- N్ phogranuloma venereum). Donovanosis was 0 ruled out too since the patient has not been in ${ }_{\bullet}$ tropical/subtropical countries and also did not have any relations with anyone who had lived $\stackrel{?}{+}$ there.

In conclusion, the present case stresses the $\frac{\vec{D}}{\mathbb{D}}$ importance of considering not only sexually $\frac{\rho}{\Phi}$ transmitted diseases, but also occupational $\varrho$ exposure in the differential diagnosis of genital granulomatous lesions.

1 Bigardi SB, Pigatto PD, Moroni P. Occupational skin gran-

2 Possick PA, Gellin GA, Key MM. Fibrous glass dermatitis. Am Indust Hyg Assoc F 1970;1:12-5.

3 Haustein UF. Arbeitsbedingte Fremdkrpergranulome de haut. Derm Mschr 1976;161:807-16.

4 Lechner W, Hartmann AA. Glasfaserinduzierte Fremdkrpergranulome. Hautarzt 1979;30:100-1.

5 Tarvainen K, Estlander T, Jolanki R, Kanerva $L$. Occupational dermatoses caused by man-made mineral fibres. Am $¥$ Contact Derm 1994;5:22-9. 